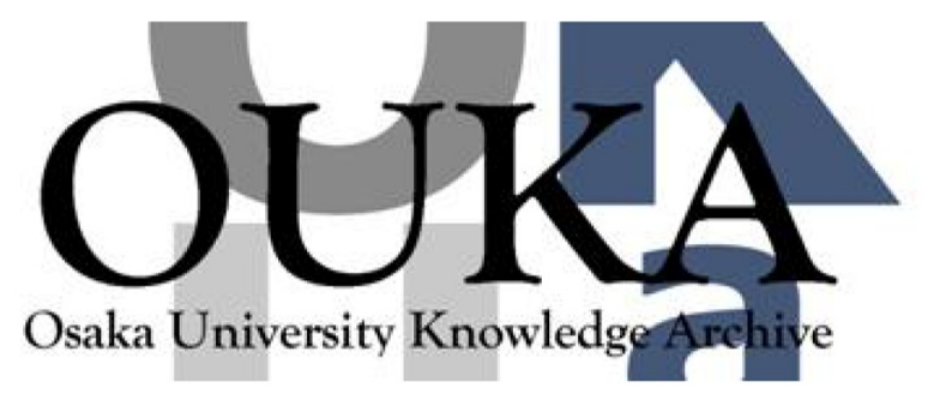

\begin{tabular}{|c|c|}
\hline Title & $\begin{array}{l}\text { Measurements of mass ablation rate and pressure } \\
\text { in planar targets irradiated by } 0.27-\mu \mathrm{m} \text { laser } \\
\text { light }\end{array}$ \\
\hline Author (s) & Boehly, T.; Tanaka, K.A.; Mochizuki, T. et al. \\
\hline Citation & $\begin{array}{l}\text { Journal of Applied Physics. 60(11) p.3840- } \\
\text { p. } 3844\end{array}$ \\
\hline Issue Date & $1986-12-01$ \\
\hline oaire:version & VoR \\
\hline URL & https://hdl. handle. net/11094/2914 \\
\hline rights & \\
\hline Note & \\
\hline
\end{tabular}

Osaka University Knowledge Archive : OUKA

https://ir. Library. osaka-u. ac. jp/

Osaka University 


\title{
Measurements of mass ablation rate and pressure in planar targets irradiated by $0.27-\mu m$ laser light
}

\author{
T. Boenly, ${ }^{\text {a) }}$ K. A. Tanaka, T. Mochizuki, and C. Yamanaka \\ Institute of Laser Engineering, Osaka University, Yamada-oka 2-6, Suita, Osaka 565, Japan
}

(Received 15 May 1986; accepted for publication 6 August 1986)

\begin{abstract}
Planar CH targets were irradiated by 400 -ps and 1 -ns pulses of $0.27-\mu \mathrm{m}$ light at intensities of $5 \times 10^{12}-10^{14} \mathrm{~W} / \mathrm{cm}^{2}$. The mass ablation rate obtained from time integrated $\mathrm{x}$-ray line emission from layered targets was found to be $\dot{m}=1.5 \times 10^{5}\left(I_{a} / 10^{13} \mathrm{~W} / \mathrm{cm}^{2}\right)^{0.5} \mathrm{~g} / \mathrm{cm}^{2} \mathrm{~s}$. Using this result and the ion blowoff velocity, the ablation pressure scaled as $P_{a}=3.9\left(I_{a} / 10^{13} \mathrm{~W} /\right.$ $\left.\mathrm{cm}^{2}\right)^{0.6} \mathrm{Mbar}$. Comparisons to similar experiments at $0.53 \mu \mathrm{m}$ indicate that the mass ablation rate and pressure scale with laser wavelength as $\lambda^{-1.4}$ and $\lambda^{-0.9}$, respectively. The intensity and wavelength scalings are found to agree with analytic predictions for spherical geometry rather than planar models. Comparisons are also made with previously reported measurements at other wavelengths and geometries.
\end{abstract}

\section{INTRODUCTION}

The rate at which mass is ablated from a directly driven, inertially confined fusion target is ultimately responsible for the compression of that target. The advantages of submicron wavelength lasers over $1 \mu \mathrm{m}$ wavelength lasers include higher ablation rate and pressure. ${ }^{1-6}$ With the decrease in laser wavelength, there is also an increase in soft $\mathrm{x}$-ray generation. ${ }^{7,8}$ The choice for optimal driver wavelength requires a trade off between the increases in ablation rate and pressure and the losses and preheating due to increased $x$-ray generation. For this choice, the scaling of ablation rate and pressure with laser parameters are needed.

Several studies have been made on the scaling of mass ablation rate for both planar ${ }^{1-5}$ and spherical targets. ${ }^{9-13}$ Most of these results are given for laser wavelengths at the harmonics of the Nd:glass laser, i.e., $1.05,0.53$, and $0.35 \mu \mathrm{m}$. Beside the absolute value of the mass ablation rate, there are also differences in the reported scaling with intensity and wavelength. At the fourth harmonic, $0.27 \mu \mathrm{m}$, little published data exist for the mass ablation rate. In Ref. 1, the scaling of the mass ablation rate with intensity and wavelength was inferred using a single datum at $0.27 \mu \mathrm{m}$. In this paper, we present measurements of the mass ablation rate for planar $\mathrm{CH}$ targets driven by $0.27-\mu \mathrm{m}$ laser light at intensities of $5 \times 10^{12}-10^{14} \mathrm{~W} / \mathrm{cm}^{2}$. In order to provide a scaling with wavelength and a normalization to previous data, we also report on mass ablation rate measurements at $0.53 \mu \mathrm{m}$. Our results indicate that the mass ablation rate scales as $I^{0.5}$ and $\lambda^{-1.4}$

An extensive study of the ablation pressure at $0.27 \mu \mathrm{m}$ has been reported. ${ }^{14-16}$ In that work, the ablation pressure was deduced from both the velocity of the accelerated target material and the speed of a shock wave within that material. Both measurements diagnose the unablated target material

\footnotetext{
") Present address: Laboratory for Laser Energetics, Univ, of Rochester, 250 E. River Rd., Rochester, NY 14623.
}

to infer the pressure on the ablation side of the target. In this paper, we present data for the ablation obtained in a different manner. By measuring the mass ablation rate and the velocity of the ablated material, we infer the ablation pressure using momentum conservation.

A comparison between our 0.53 and $0.27 \mu \mathrm{m}$ data indicates that the ablation pressure scales as $I^{0.6}$ and $\lambda^{-0.9}$. We find that within the expected errors in normalization, our data are consistent with other reports on mass ablation rate ${ }^{1}$ and pressure ${ }^{16}$ for $0.27 \mu \mathrm{m}$ at around $10^{14} \mathrm{~W} / \mathrm{cm}^{2}$. Our study serves to extend the range of intensities for which the mass ablation rate is measured and provides an additional measure of the ablation pressure for $0.27 \mu \mathrm{m}$ at intensities not yet reported.

\section{EXPERIMENTAL CONDITHONS}

Planar targets were irradiated with 400-ps and 1-ns pulses of $0.27-\mu \mathrm{m}$ laser light at intensities of $5 \times 10^{12}-10^{14}$ $\mathrm{W} / \mathrm{cm}^{2}$. The experiments were carried out using the frequency quadrupled beam of the Gekko IV laser at the Institute of Laser Engineering. ${ }^{8}$ Typical laser energies of 3-6 J were focused onto spots of $100-300 \mu \mathrm{m}$ diameter using an f/8 quartz lens.

The targets were massive aluminum foils with overcoatings of $0-3 \mu \mathrm{m} \mathrm{CH}$. The mass ablation rate was determined by observing the time integrated $x$-ray emission from the aluminum substrates as the thickness of the $\mathrm{CH}$ coating was varied. The line emission was measured using an $x$-ray crystal spectrometer, which employed a thallium-acid-phthalate (TIAP) crystal. The image of the $x$-ray emission was recorded using an $x$-ray pinhole camera with a $25-\mu \mathrm{m}$ beryllium filter and a $10-\mu \mathrm{m}$ pinhole. Both the crystal spectrometer and the pinhole camera used Kodak 2494 RAR base film. The spectral resolution of the crystal spectrometer for the spot sizes used was $\lambda / \Delta \lambda=350$ and the spatial resolution of the pinhole camera was about $12 \mu \mathrm{m}$. The spectrometer and pinhole camera viewed the front (laser side) of the target at 

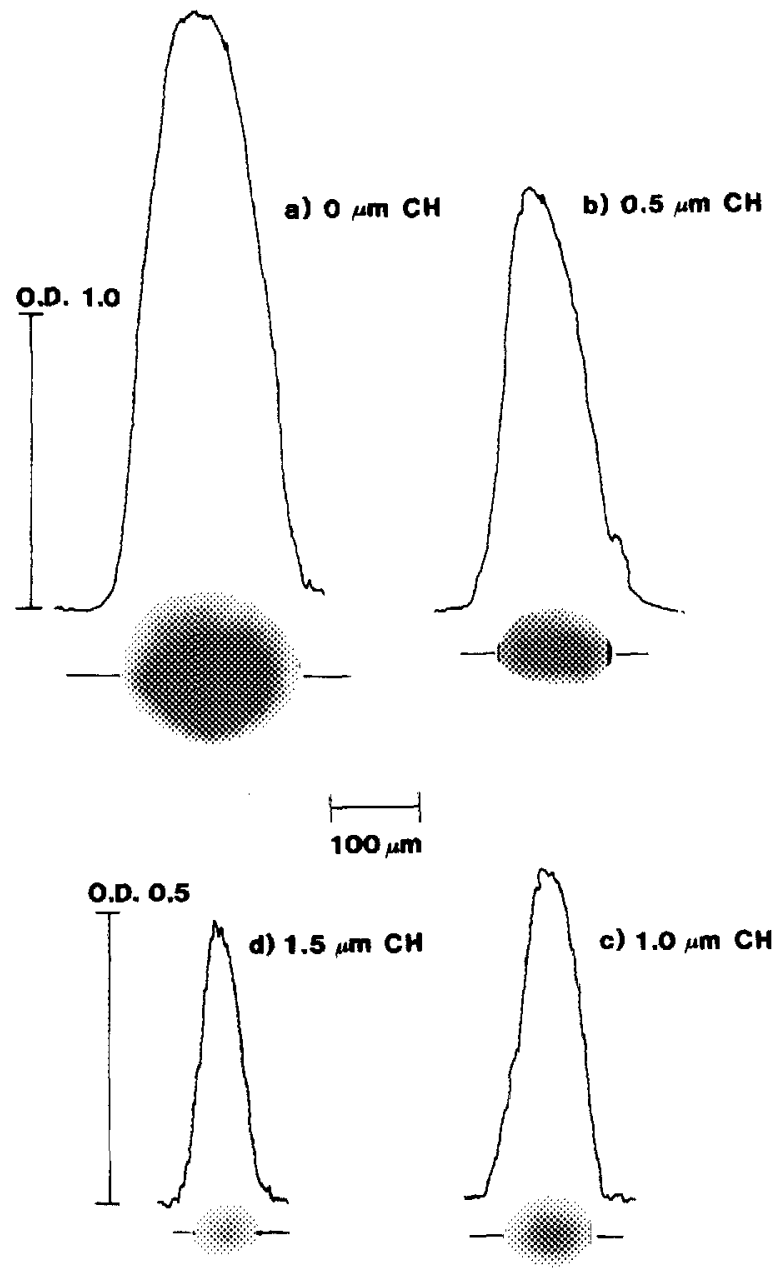

FIG. 1. The $x$-ray pinhole camera images and densitometer traces of targets with (a) $0 \mu \mathrm{m}$, (b) $0.5 \mu \mathrm{m}$, (c) $1.0 \mu \mathrm{m}$, and (d) $1.5-\mu \mathrm{m}$ overcoating of $\mathrm{CH}$. The size of the $\mathrm{x}$-ray emission at various coating thickness is analogous to isointensity contours in the intensity distribution of the laser spot.

opposing $45^{\circ}$ angles. The velocity of blowoff plasma was monitored using a Faraday cup charge collector located at $10^{\circ}$ from the target normal. All targets were irradiated at normal incidence.

The pinhole camera was used to monitor the intensity distribution of the laser. Shown in Fig. 1 are the pinhole camera images of the $x$-ray emission from the aluminum substrates of various targets. Figures $1(\mathrm{a})-(\mathrm{d})$ are the images of the emission from targets with $0,0.5,1.0$, and $1.5 \mu \mathrm{m}$ of $\mathrm{CH}$ overcoating, respectively. The laser energy and focal position were the same for all of the shots in Fig. 1. In order to define an intensity, we assumed that the radial intensity profile of the beam is gaussian and the peak intensity $\left(I_{0}\right)$ is defined by the laser power and the area enclosed by the $I_{0} / e$ radius.

For a given pulse duration, the local penetration depth is proportional to the intensity distribution of the laser spot. The onset of $\mathrm{x}$-ray emission from the aluminum substrate will occur only if the local intensity is above the threshold needed to burn through the $\mathrm{CH}$ overcoating. In Fig. 1, the images from the various targets can therefore represent isointensity contours in the laser spot. By assuming that the instantaneous mass ablation rate depends upon the intensity as $I^{0.5}$, we calculated local penetration depth of a temporally and spatially Gaussian pulse into the target. From that depth, we calculated the maximum diameter of the $x$-ray emission from the aluminum substrate. We found that the rate of change of the observed diameter with $\mathrm{CH}$ thickness was fit by this estimate. We conclude that, within an error of $\pm 20 \%$, the spots can be fit by spatially Gaussian forms.

The images in Fig. 1 demonstrate that the intensity distribution of the laser spot is center peaked and is free of hot spots which could yield misleading results for the penetration depth. For much larger spot sizes, the aberrations of the beam caused a ring structure composed of several high intensity regions. Although not apparent in the images from bare aluminum targets, these hot spots become more pronounced as the $\mathrm{CH}$ overcoating thickness was increased. We restricted our mass ablation measurement to spots which could be characterized by centrally peaked distributions. This limited the range in spot diameters to between 100 and $300 \mu \mathrm{m}$.

\section{MASS ABLATION RATE}

The penetration of the heat front into the solid is accompanied by heating and ionization of the target material. Target mass which is ablated enters the high pressure region of the plasma and is accelerated outward. In the determination of mass ablation rate, we are ultimately interested in the total amount of mass which is ablated during the laser pulse. As in the method reported in Ref. 2, the penetration of the heat/ ionization front is observed using the time integrated $x$-ray emission from the target substrates. If the heat front is steep, the line emission from highly ionized atoms is indicative of ablation. The penetration depth is then a measure of the total mass which has been ablated. Nonlocal effects which can cause preheating and misleading high penetration depths for $1-\mu \mathrm{m}$ experiments ${ }^{10}$ are diminished for submicron wavelength lasers. ${ }^{.1}$

Figure 2 shows the intensity of the H-like Al line at 1.73 $\mathrm{keV}$ versus the overcoating of $\mathrm{CH}$. The results for pulse durations of $400 \mathrm{ps}$ (triangles) and $1 \mathrm{~ns}$ (squares) are shown. The ordinate is the line intensity normalized to the intensity from a bare $(0 \mu \mathrm{m} \mathrm{CH})$ target and to the laser energy for each shot. The laser energy in each series of shots at a given intensity varied by less than $20 \%$ in order to provide an approximately constant intensity for a given curve. Since the $x$-ray emission from the aluminum is proportional to the thermal energy which has penetrated the $\mathrm{CH}$ coating, these curves represent the penetration of the heat front into the targets. The intensity dependence of mass ablation rate can be seen by comparing the depth of penetration at the same pulse duration.

The mass ablation rate is given as: $\dot{m}=C \rho_{0} d_{x} / t_{p}$, where $\rho_{0} / d_{x}$ is the ablated areal mass given by the target density $\rho_{0}$ and the penetration depth $d_{x}$, and $t_{p}$ is the full width half maximum ( $F W H M$ ) time of the laser pulse. A correction factor ${ }^{4} C=(4 \ln 2 / \pi \alpha)^{0.5}$ accounts for an $I^{\alpha}$ dependence of the mass ablation rate during a temporally gaussian laser pulse. The penetration depth $d_{x}$ is determined from the overcoat thickness which attenuates the aluminum line emission by an order of magnitude. This somewhat conservative estimate was chosen in Ref. 10 in order not to in- 


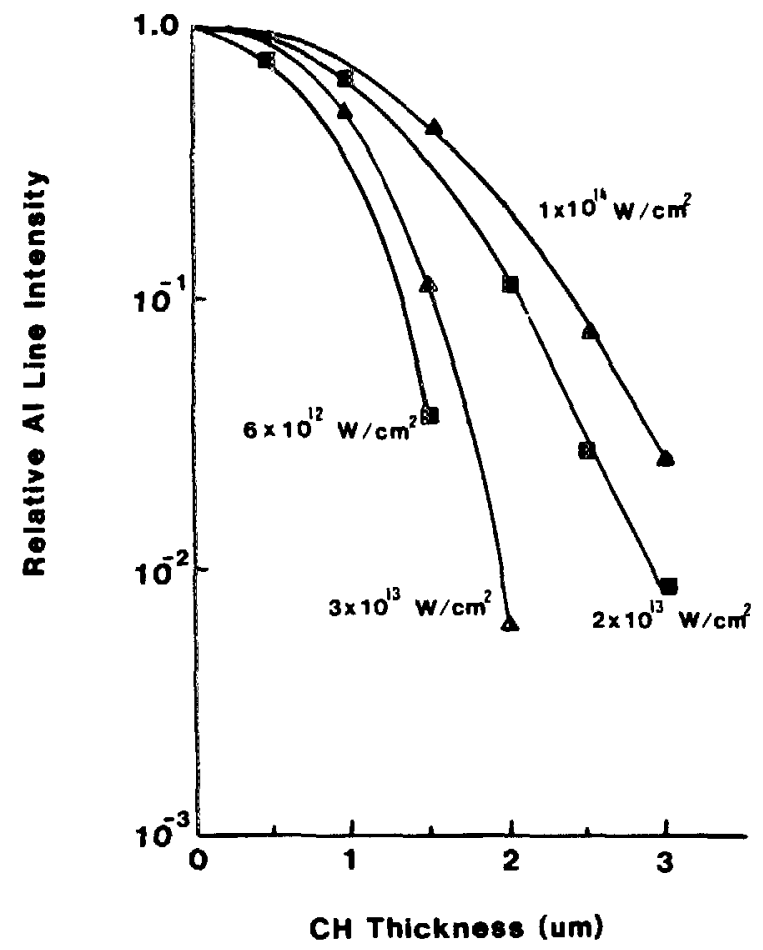

FIG. 2. The intensity of the $\mathrm{Al} \mathrm{x}$-ray line from the aluminum substrates as a function of the $\mathrm{CH}$ overcoating for 400-ps (triangles) and l-ns (squares) pulses. The intensity dependence of the penetration depth can be noted by comparing the curves at the same pulse duration.

clude heat material ahead of the actual ablation front.

Since the $x$-ray spectrometer data are spatially integrated, an additional definition for the peak depth was obtained by normalizing the $x$-ray line intensity data to the $x$-ray emission source size. The source size was obtained from the $x$-ray pinhole camera images. This normalization includes the effect of reduced emission size, which accompanies thicker overcoatings. The burnthrough curves obtained in this manner were flat, i.e., an approximately constant value is observed until the penetration depth is reached. At that point, the intensity drops more steeply than shown in Fig. 2. The penetration depth inferred by this manner was $20 \%$ higher than determined from the curves in Fig. 2.

As a final check, a third definition was also used. The optical density at the center of the spectrally integrated pinholes images (see Fig. 1) were plotted versus the $\mathrm{CH}$ coating. This measurement gave a penetration depth which was $20 \%$ lower than the first deinnition. The lower inferred penetration depths are a result of the reduction of the higher energy lines which are included in the pinhole camera images. For increasing $\mathrm{CH}$ overcoating, these higher energy lines decay more rapidly than the Lyman $-\alpha$ line. The error in the mass ablation rate values is given by these measurements and the experimental uncertainty in line intensity determination.

Figure 3 is a plot of the mass ablation rate versus the absorbed laser intensity. The experimental data for $400 \mathrm{ps}$ and $1 \mathrm{~ns}$ are shown as triangles and squares, respectively. Also included in Fig. 3 is the datum (closed circle) for the mass ablation rate for a planar target irradiated by a 170 -ps

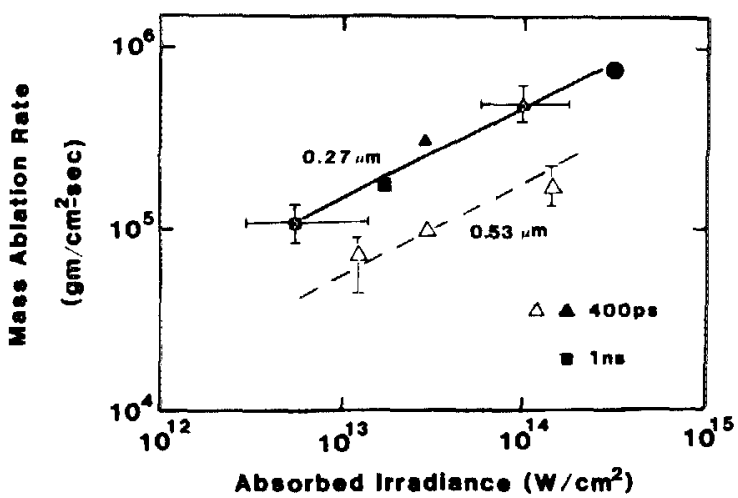

FIG. 3. The mass ablation rate vs absorbed laser irradiance for $0.27 \mu \mathrm{m}$ (closed points) and $0.53-\mu \mathrm{m}$ (open points) light. Data at $400 \mathrm{ps}$ (triangles) and $1 \mathrm{~ns}$ (squares) are shown. Also shown is the datum (closed circle) from Ref. 1 for $0.27 \mu \mathrm{m}$.

pulse of $0.27-\mu \mathrm{m}$ light. ' The scaling suggested by our data is

$$
\dot{m}=1.5 \times 10^{5}\left(I_{a} / 10^{13} \mathrm{~W} / \mathrm{cm}^{2}\right)^{0.5} \mathrm{~g} / \mathrm{cm}^{2} \mathrm{~s},
$$

where $I_{a}$ is the absorbed laser irradiance. This scaling has incorporated our data at both $400 \mathrm{ps}$ and $1 \mathrm{~ns}$. If the interaction is steady state, we expect that differences in pulse duration will not affect the resultant scalings. As will be shown later, the ion current traces indicate that the interaction is quasisteady even for the 400-ps pulse width. The analysis of Ref. 17 also suggests that for our experimental conditions, steady state should be reached before the peak of the pulse. The agreement of the datum from Ref. 1 ( $170 \mathrm{ps)}$ and our data also support our contention that, for $\lambda=0.27 \mu \mathrm{m}$, the pulse width does not significantly affect the scaling of mass ablation rate.

The results for similar targets irradiated by $0.53-\mu \mathrm{m}$ light are shown in Fig. 3 as open triangles and a dashed line. The results at lower intensities have a greater error because the targets were not optimized for the small penetration depths associated with $0.53 \mu \mathrm{m}$ light and the burnthrough curves were not as well resolved. The dashed line is forced fit to the same scaling $\left(I^{0.5}\right)$ as the $0.27-\mu \mathrm{m}$ data and is a factor of 3 lower. We forced the fit at $0.53 \mu \mathrm{m}$ in order to determine the scaling of mass ablation rate. The comparison of the data indicates a scaling with laser wavelength as $\lambda^{-1.4}$. The poor fit to the $0.53-\mu \mathrm{m}$ data results from both the errors at lower intensity and the effects of lateral transport which have been noted at higher intensities. ${ }^{4}$

\section{ABLATION PRESSURE}

In the assumption of steady state planar geometry, the ablation pressure can be deduced directly from the conservation of momentum and is given by the mass ablation rate and the flow velocity. In that assumption, the ablation pressure is $P_{a}=\dot{m} \bar{v}$, where $\bar{v}$ is the average asymptotic ion velocity. The expansion hydrodynamics of planar targets are determined by the laser spot size $d_{0}$ and a characteristic expansion distance which is given by the sound speed $c_{s}$ and the pulse duration $t_{p}$. Plasmas from plane targets irradiated by laser spots with $d_{0} / c_{s} t_{p}<1$ will exhibit spherical expansions. ${ }^{18}$ The effects of this spherical expansion can be accounted for 


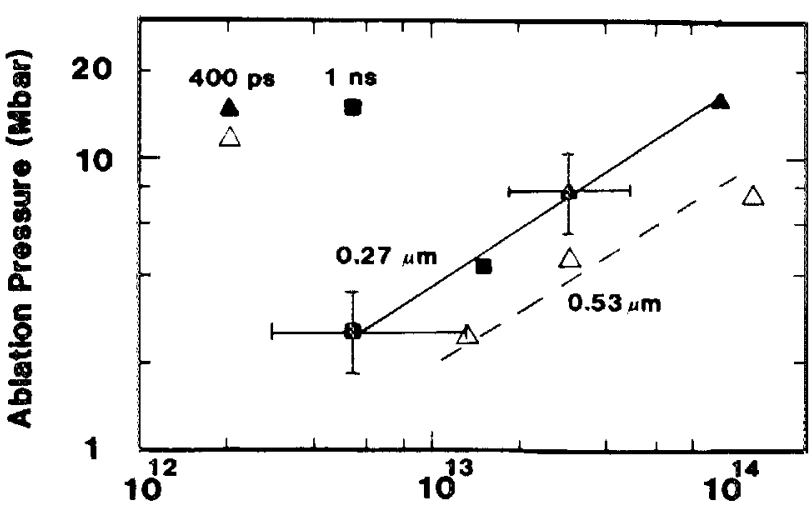

Absorbed Irradiance $\left(W / \mathrm{cm}^{2}\right)$

FIG. 4. The peak ablation pressure vs the absorbed laser irradiance for 400 ps (triangles) and 1-ns (squares) pulses at $0.27 \mu \mathrm{m}$ (closed points) and $0.53 \mu \mathrm{m}$ (open points). The ablation pressure is determined form the mass ablation rate and the ion exhaust velocity.

by a correction factor whose value has been determined from simulations to be between $0.5^{9}$ and $0.7^{11}$. This factor relates the asymptotic ion velocity and mass ablation rate to the ablation pressure in the same manner as is done in the experiment.

The above analysis requires an average ion velocity which we found from the charge collector signal. The current trace from the charge collector was distinctly peaked as compared to that expected from an isothermal expansion. The current trace from an isothermal expansion will have a $\Delta t / t=1.7$, where $\Delta t$ is the full width at half maximum (FWHM) and $t$ is the time at peak current. The experimental traces from the 400 -ps and 1 -ns pulses $\Delta t / t$ was $\simeq 0.7$ and $\simeq 0.5$, respectively. This is indicative of quasisteady ablation, hence the mean velocity of the expansion is best represented by the peak of the current trace. The experimental ion velocity for $0.27-\mu \mathrm{m}$ light is given by the expression:

$$
v_{p}=3.7 \times 10^{7}\left(I_{a} / 10^{13} \mathrm{~W} / \mathrm{cm}^{2}\right)^{0.1} \mathrm{~cm} / \mathrm{s} .
$$

Figure 4 shows the results for the ablation pressure derived from the mass ablation rate data in Fig. 3 and the exhaust velocity given in Eq. (2). A power law dependence fit to the pressure data yields

$$
P_{a}=3.9\left(I_{a} / 10^{13} \mathrm{~W} / \mathrm{cm}^{2}\right)^{0.6} \mathrm{Mbar} \text {. }
$$

Shown as open points and a dashed line in Fig. 4 are the results for $0.53-\mu \mathrm{m}$ irradiation. By comparing the pressure for $0.53-\mu \mathrm{m}$ light obtained in the same manner, we infer a scaling with laser wavelength of $\lambda^{-0.9}$.

\section{DISCUSS:ON}

The reported experimental results for mass ablation rate appear to exhibit two different scalings with absorbed laser intensity either $I^{0.5}$ (Refs. 2, 3, 5, 8, 9,11,12) or $I^{0.33}$ (Refs. $1,4)$. While both planar and spherical targets have exhibited the former scaling, only planar targets were observed to follow the latter. One-dimensional analytical models predict intensity scalings of $I^{0.5}$ for spherical geometry ${ }^{6}$ and $I^{1 / 3}$ for planar geometry. ${ }^{18}$ However, the planar analytical model has treated the case of laser energy deposition at the critical surface only. The authors of Ref. 5 used a simple approximation which accounts for inverse bremsstrahlung absorption to explain their planar results which exhibited a $I^{0.5}$ dependence.

While it is difficult to conclude from the scaling of the mass ablation rate, we expect that our small spots are dominated by spherical expansion effects. ${ }^{19}$ The agreement in the results of Refs. 1 and 4 and the planar analytic model may be a result of the manner in which the laser light is absorbed rather than that of the geometry, since a truly planar experiment ${ }^{20}$ at $1.06 \mu \mathrm{m}$ exhibited an $\dot{m}$ scaling of $I^{0.5}$.

Our mass ablation results are in good agreement with the datum at $\simeq 3 \times 10^{14} \mathrm{~W} / \mathrm{cm}^{2}$ for a $0.27-\mu \mathrm{m}$ planar experiment ${ }^{1}$ and the intensity scaling ${ }^{13}$ reported for $0.27-\mu \mathrm{m}$ spherical targets at $0.27 \mu \mathrm{m}$. The different intensity scaling reported for planar targets ${ }^{1.4}$ and longer wavelength light is not understood. These differences may be ascribed to different experimental conditions between laboratories, e.g., the intensity distribution of the lasers or the manner in which the intensity is varied.

If the value of the mass ablation rate at a given intensity is directly compared to the data at other wavelengths, the mass ablation rate appears to be independent of laser wavelength. This observation led us to perform similar experiments at $0.53 \mu \mathrm{m}$ to verify the dependence of the mass ablation rate on laser wavelength. This comparison yielded a scaling of $\lambda^{-1.4}$ which is about that predicted by most analytic models. This indicates that, for $\mathrm{CH}$ targets, there are not appreciable losses due to enhanced $x$-ray generation at $0.27 \mu \mathrm{m}$. The reason for the apparent error in the comparison of the absolute values of mass ablation rates from various laboratories is still unresolved.

The pressure is observed to scale as $I^{0.6}$ and $\lambda^{-0.9}$. The slower dependence on wavelength as compared to the mass ablation rate is a result of the increase in blowoff velocity for the $0.53-\mu \mathrm{m}$ light. The scaling values are in agreement with analytic and numerical predictions in spherical geometry. The extrapolation of our results for the ablation pressure to the range reported in Ref. 16 show very good agreement between the two experiments at $0.27 \mu \mathrm{m}$. As indicated in that work, numerical simulations overestimated the experimental ablation pressure at high intensities. This was cited as a result of lateral transport which caused significant energy loss from the laser spot. Since this effect was not observed for intensities below $10^{14} \mathrm{~W} / \mathrm{cm}^{2}$ (Ref. 16), we expect that our experiments do not experience such losses.

In contrast to Ref. 16, hydrocodes with a flux limiter typically underestimate the experimental mass ablation rate (penetration depth) ) ${ }^{9.10}$ This indicates the coupling of laser energy to the target is more efficient than predicted. Thus, rather than inferring losses due to lateral transport, we conclude that the numerical description for thermal conduction is insufficient. Currently, we are attempting to include a nonlocal treatment for the thermal transport in our hydrocode. The conclusions of Ref. 16 may be a result of the comparison of a basically spherical experiment to a planar simulation. Our results indicate that spherical geometry may be a better approximation. 


\section{CONCLUSIONS}

We have measured the ablation rate in planar targets irradiated by $0.27-\mu \mathrm{m}$ laser light. The mass ablation rate scaled with intensity and wavelength as $I^{0.5}$ and $\lambda^{-1.4}$. The results which were presented were found to agree with previous data at $0.27 \mu \mathrm{m}$ and have served to extend the range of intensities at which the mass ablation rate has been measured.

Our results have also provided a third manner in which the ablation pressure is measured at $0.27 \mu \mathrm{m}$. The pressure was found to scale as $I^{0.6}$ and $\lambda^{-0.9}$. Although we could not exactly simulate this data, we found the scalings for both the ablation rate and pressure were in good agreement with those predicted by numerical codes and analytic models which account for a delocalized deposition of the laser energy. We found no conclusive evidence supporting the presence of lateral transport in our experiments at $0.27 \mu \mathrm{m}$. This data should prove useful as a reference for simulations aimed at understanding thermal transport at very short laser wavelengths.

\section{ACKNOWLEDGMENTS}

The authors would like to thank Dr. Yabe and Dr. Nishihara for helpful discussions. The technical assistance of M. Mineo, R. Kodama, N. Doi, and H. Nakano is gratefully acknowledged. One author (T.B.) would like to thank the Laser Society of Japan for the support of his stay at ILE.

'F. Amiranoff, R. Fabbro, E. Fabre, C. Garban, J. Virmont, and M. Weinfeld, Phys. Rev. Lett. 43, 522 (1979); R. Fabbro, E. Fabre, F. Amiranoff, C. Garban-Labaune, J. Virmont, M. Weinfeld, and C. E. Max, Phys. Rev. A 26, 2289 (1982).

${ }^{2}$ B. Yalakobi, T. Boehly, P. Bourke, Y. Conturie, R. S. Craxton, J. Delettrez, J. M. Forsyth, R. D. Frankel, L. M. Goldman, R. L. McCrory, M. C. Richardson, W. Seka, D. Shvarts, and J. M. Soures, Opt. Commun. 39, 175 (1981).
'H. Nishimura, H. Azechi, K. Yamada, A. Tamura, Y. Inada, F. Matsuoka, H. Hamada, Y. Suzuki, S. Nakai, and C. Yamanaka, Phys. Rev. A. 23, 2011 (1981)

${ }^{4}$ M. H. Key, W. T. Toner, T. J. Goldsack, J. D. Kilkenny, S. A. Veats, P. F. Cunningham, and C. L. S. Lewis, Phys. Fluids 7, 2011 (1983).

${ }^{5}$ B. Meyer and G. Thiell, Phys. Fluids 27, 302 (1984).

${ }^{6}$ C. E. Max, C. F. McKee, and W. C. Mead, Phys. Rev. Lett. 45, 28 (1980). 'W. C. Mead, E. M. Campbell, K. G. Estabrook, R. E. Turner, W. L. Kruer, P. H. Y. Lee, B. Pruit, V. C. Rupert, K. G. Tirsell, G. L. Stradling, F. Ze, C. E. Max, and M. D. Rosen, Phys. Rev. Lett 47, 1289 (1981); B. Yalakobi, P. Bourke, Y. Conturie, J. Delettrez, J. M. Forsyth, R. D. Frankel, L. M. Goldman, R. L. McCrory, W. Seka, and J. M. Soures, Opt. Commun. 38, 196 (1981).

${ }^{8}$ R. Kodama, K. Okada, N. Ikeda, M. Mineo, K. A. Tanaka, M. Mochizuki, and C. Yamanaka, J. Appl. Phys. 59, 3050 (1986).

'T. J. Goldsack, J. D. Kilkenny, B. J. MacGowan, P. F. Cunningham, C. L. S. Lewis, M. H. Key, and P. T. Rumsby, Phys. Fluids 25, 1634 (1982).

${ }^{10}$ B. Yalakobi, J. Delettrez, L. M. Goldman, R. L. McCrory, R. Majoribanks, M. C. Richardson, D. Shvarts, S. Skupsky, J. M. Soures, C. Verdon, D. M. Villeneuve, T. Boehly, R. Hutchinson, and S. Letzring, Phys. Fluids 27, 516 (1984).

"'B. Yalakobi, O. Barnouin, J. Delettrez, L. M. Goldman, R. Majoribanks, R. L. McCrory, M. C. Richardson, and J. M. Soures, J. Appl. Phys. 57, 4354 (1984).

${ }^{12}$ T. Yamanaka, T. Mochizuki, H. Azechi, H. Nishimura, M. Yamanaka, N. Miyanaga, H. Niki, E. Fujiwara, H. Shiraga, K. Okada, S. Sakabe, Y. Kato, Y. Kitagawa, M. Nakatsuka, K. Mima, N. Nishihara, T. Yabe, S. Nakai, and C. Yamanaka, Lasers Part. Beams 4, Part 1, 43 (1985).

${ }^{13}$ E. Fabre, C. LaBaune, R. Fabbro, B. Faral, A. Michard, H. Pepin, A. Poquerusse, J. Virmont, F. Briand, J. Briand, P. Mora, J. F. Luciani, R. Pellat, H. Baldis, F. Cottet, and J. P. Romain, Proc. IAEA Tenth Annual Conf. on Plasma Physics and Controlled Nuclear Fusion Research, London, UK, 12-14 Sept. 1984, IAEA-CN-44/B-III-4.

${ }^{14}$ R. Fabbro, B. Faral, F. Cottet, and J. P. Romain, J. Appl, Phys. 56, 3204 (1984).

${ }^{15}$ H. Pepin, R. Fabbro, B. Faral, F. Amiranoff, J. Virmont, F. Cottet, and J. P. Romain, Phys. Fluids 28, 3393 (1985).

${ }^{16}$ R. Fabbro, B. Faral, J. Virmont, F. Cottet, and J. P. Romain, and H. Pepin, Phys. Fluids 28, 3414 (1985).

${ }^{17}$ R. Fabbro, C. E. Max, and E. Fabre, Phys. Fluids 28, 1463 (1985).

${ }^{18}$ M. Herbst and J. Grun, Phys. Fluids 24, 1917 (1981).

${ }^{19} \mathrm{G}$. Matzen and R. L. Morse, Phys. Fluids 22, 654 (1979).

${ }^{20}$ J. Grun, S. P. Obenschain, B. H. Ripin, R. R. Whitlock, E. A. McLean, J. Gardner, M. J. Herbst, and J. A. Stamper, Phys. Fluids 26, 588 (1983). 